\title{
Clinical efficacy, timing, and outcomes of ERCP for management of bile duct leaks: a nationwide cohort study
}

\section{(ㄷ)(요 $\odot$}

\section{Authors}

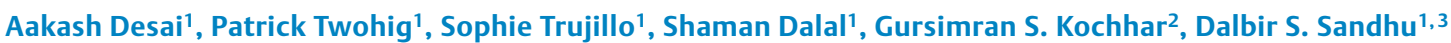

\section{Institutions}

1 Department of Internal Medicine, MetroHealth Medical Center, Cleveland, Ohio, United States

2 Division of Gastroenterology, Hepatology \& Nutrition, Allegheny Health Network, Pittsburgh, Pennsylvania, United States

3 Division of Gastroenterology, Hepatology \& Nutrition, Digestive Disease and Surgery Institute, Cleveland Clinic, Cleveland, Ohio, United States

submitted 2.8 .2020

accepted after revision 22.10 .2020

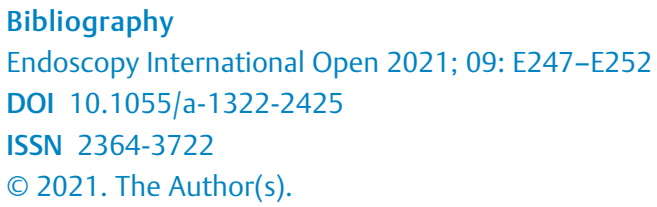

This is an open access article published by Thieme under the terms of the Creative Commons Attribution-NonDerivative-NonCommercial License, permitting copying and reproduction so long as the original work is given appropriate credit. Contents may not be used for commecial purposes, or adapted, remixed, transformed or built upon. (https://creativecommons.org/licenses/by-nc-nd/4.0/)

Georg Thieme Verlag KG, Rüdigerstraße 14,

70469 Stuttgart, Germany

Corresponding author

Dalbir S. Sandhu, MD, Director of Endoscopy, Digestive

Disease and Surgery Institute, Cleveland Clinic, Akron

General, 1 Akron General Ave, Akron, OH. 44307, USA

Fax: +1-319-356-1025

drdalbir@gmail.com

\section{ABSTRACT}

Background and study aims Endoscopic retrograde cholangiopancreatography (ERCP) can safely and effectively manage postsurgical or traumatic bile duct leaks (BDLs). Standardized guidelines are lacking regarding effective management of BDLs. Our aim was to evaluate the efficacy, clinical outcomes, and complications of different ERCP techniques and intervention timing using a nationwide database.

Patients and methods We performed a retrospective analysis of the IBM Explorys database (1999-2019), a pooled, national, de-identified clinical database of over 64 million unique patients across the United States. ERCP timing after BDL was classified as emergent ( $<1$ day), urgent (1-3 days) or expectant ( $>3$ days). ERCP technique was classified into sphincterotomy, stent or combination therapy. ERCP complications were defined as pancreatitis, duodenal perforation, duodenal hemorrhage, and ascending cholangitis within 7 days of the procedure.

Results Expectant ERCP had a decreased risk of adverse events (AEs) compared to emergent and urgent ERCP ( $P=$ $0.004)$. Rehospitalization rates also were lower in expectant ERCP $(P<0.001)$. Patients with COPD were more likely to have an $A E$ if the ERCP was performed emergently compared to expectantly $(P=0.002)$. Combination therapy had a lower rate of ERCP failure compared to placement of a biliary stent $(P=0.02)$. There was no statistically significant difference in rates of ERCP failure between biliary stent and sphincterotomy $(P=0.06)$ or sphincterotomy and combination therapy $(P=0.74)$.

Conclusion Our study suggests that ERCP does not need to be performed emergently or urgently for management of BDLs. Combination therapy is superior to stenting but not sphincterotomy; however, future prospective studies are needed to validate these findings.

\section{Introduction}

Bile duct leaks (BDLs) can arise as a complication of surgery or trauma to the biliary system. Although rare, common surgeries that can cause BDLs include cholecystectomy, hepatectomy, liver transplant, and the Whipple procedure. BDLs lead to accu- mulation of bile in the abdominal cavity, which can cause symptoms such as pain, nausea, vomiting, fever, and jaundice. Early diagnosis is essential as unrecognized BDLs can lead to sepsis and death. Endoscopic retrograde cholangiopancreatography (ERCP) can safely and effectively manage bile duct leaks [1] The goal of treatment includes creating a low-pressure system 
to promote flow to the duodenum. This, in turn, can help to decrease a biliary leak and thus create an environment in which the bile duct can heal [1].

Techniques that are commonly used include bile duct sphincterotomy, bile duct stenting, or a combination of both $[2,3]$. Recent studies have evaluated the clinical efficacy and outcomes of these techniques, but the results have been varied and controversial [4-6]. Studies also have assessed optimal timing of ERCP [7-11], typically referencing literature on the timing of endoscopy for gastrointestinal bleeding [12-14]. There is a lack of standardized guidelines regarding the effective management of BDLs. The American Society for Gastrointestinal Endoscopy (ASGE) recommends biliary decompression by either sphincterotomy alone or nasobiliary drain placement, with or without sphincterotomy [15]. However, the European Society for Gastrointestinal Endoscopy (ESGE) recommends biliary stent placement only without sphincterotomy to avoid both short- and long-term adverse events (AEs) [16].

In addition to the variation seen in endoscopic technique for repairing BDLs, the timing of intervention varies between different centers. Prior studies have divided the timing of intervention into emergent ( $<1$ day), urgent ( $2-3$ days), and expectant (>3 days) [4]. There are no standardized guidelines regarding the optimal timing of intervention for BDLs [1,4]. The primary aim of our study was to evaluate the efficacy, complications, and clinical outcomes of ERCP timing for management of BDLs. The secondary aim was to assess the impact of BDL etiology on ERCP timing and outcomes and to evaluate the efficacy of different ERCP techniques.

\section{Patients and methods}

\section{Database}

This was a retrospective cohort study using Explorys [17], a prospectively maintained electronic medical record (EMR) dataset (IBM Corporation, Somers, New York, United States). Explorys is a multi-institution, de-identified EMR database with over 70 million covered patient lives spanning from 1999 to 2020. De-identified data from virtual healthcare systems (EPIC, Amalga, Eclipsys, etc.) are standardized and normalized by the Explorys web-based platform. Specific diagnoses, procedures, and medications are mapped into the systematized nomenclature of medicine clinical terms (SNOMED-CT). Each unique search allows users to further specify associated medications, comorbidities, demographics, and vital signs. All data are live and updated once every 24 hours [1]. Each participating organization has access to a password-protected web-based application (https://popex.explorys.com). Explorys is a Health Insurance Portability and Accountability Act (HIPAA)- and Health Information Technology for Economic and Clinical Health Act (HITECH)-compliant platform, and institutional review board approval for its use is not required. This study was reported according to the Strengthening the Reporting of Observational Studies in Epidemiology (STROBE) guidelines for observational cohort studies [2].

\section{Study design}

Patients with BDL were identified using the diagnosis of "injury of bile duct: in the Explorys database. Etiology of BDL was categorized into cholecystectomy, trauma, and liver transplant. Patients who underwent ERCP were identified using the procedure code of "endoscopic retrograde cholangiopancreatography." The Explorys database allows for establishing temporal relationships between factors such as certain diagnoses, procedures, and medications to ensure they occurred after a specific event. Using this function, we were able to categorize the timing of ERCP after BDL and subsequently identify AEs and ERCP complications. To avoid duplication of patients who leave and enter the healthcare system, Explorys uses a patient matching engine, which ensures that a patient is captured only once throughout their life within the health platform. This temporal relationship methodology was applied to our primary and secondary analysis as follows.

ERCP timing was defined as emergent if it occurred within 1 day, urgent if it occurred in 2 to 3 days, and expectant if it occurred 3 days after the BDL. AEs included one of the following if they occurred within 2 weeks of ERCP: intubation, invasive hemodynamic monitoring, central venous cannula insertion, hemodialysis, transfusion of blood product, insertion of tracheostomy, total parenteral nutrition, abscess of liver, drainage of subphrenic abscess, shock, sepsis, or pneumonia. These specific complications were included to maintain consistency with prior research evaluating BDLs [4]. Post-ERCP complications included one of the following, if they occurred within 7 days of ERCP: pancreatitis, ascending cholangitis, duodenal perforation, or duodenal hemorrhage. Rehospitalization rates included diagnosis of one of the following within 3 months: bile peritonitis, abdominal abscess, or persistent bile duct leak. Patients with persistent BDL were identified as those who had a repeat diagnosis of bile duct injury within 3 months of ERCP.

Type of ERCP intervention was categorized into sphincterotomy, bile stent placement, and combination of sphincterotomy and bile stent placement. ERCP failure was defined as need for salvage biliary surgery, which included percutaneous transhepatic biliary drainage or bile duct repair.

Subgroup analysis was attempted based on the etiology of the BDL as mentioned above. Patients who had cholecystectomy-related BDL were included in the study.

Limited analysis could be performed on patients with traumarelated BDL. For HIPAA-compliant statistical de-identification purposes, the Explorys database does not allow reporting on sample sizes less than 10 and all population counts above 10 are rounded to the nearest 10 . Liver transplant-related BDL was excluded from the analysis due to extremely small sample size.

\section{Statistical analysis}

The proportion of patients based on ERCP timing, procedure type, AEs, ERCP failure, and post-ERCP complications were reported as percentages. Descriptive characterization of baseline demographics was done in patients who developed AEs after 
emergent, urgent, and expectant ERCP. A chi-square test of independence was used to analyze the relationship between ERCP timing and AEs, ERCP timing and ERCP complications, and ERCP technique and failure rates. Social Science Statistic, an online statistical tool audited for accuracy compared to SPSS, was used to calculate the chi-squared test statistic.

\section{Results}

There were 1260 patients with BDL included in the study. The etiology of BDL was cholecystectomy (52.38\%), trauma (25.4\%), and liver transplant (1.59\%). Baseline demographics for patients who underwent emergent, urgent, and expectant ERCP can be found in $>$ Table 1 . There was no statistical difference based on demographic parameters between the three groups.

\section{Impact of ERCP timing}

Six hundred patients (47.62\%) underwent ERCP after a BDL; 200 (33.33\%) were done emergently, 100 (16.67\%) were done urgently, and 300 (50\%) were done expectantly. The rate of AEs in patients who underwent ERCP emergently or urgently was higher compared to patients who underwent the procedure expectantly ( $30 \%$ vs $16.67 \%, P=0.004$ ). The rate of ERCP complication between patients who had the procedure done emergently compared to expectantly ( $5 \%$ vs $6.67 \%, P=0.44$ ) was not statistically significant. Rehospitalization rates were higher in emergent and urgent ERCP compared to expectant (40\% vs $40 \%$ vs $20 \%, P<0.001$ ) ( Fig. 1). Patients with COPD were more likely to have an AE if the ERCP was performed emergently compared to expectant management ( $17 \%$ vs $0 \%, P=0.002$ ). Other demographics parameters were not statistically significant for patients based on procedure timing ( $\downarrow$ Table 2 ).

\section{Impact of BDL etiology}

\section{Cholecystectomy}

Six hundred sixty patients with cholecystectomy developed BDL and 420 patients (63.64\%) underwent ERCP. ERCP was performed emergently in 140 patients (33.3\%), urgently in 70 patients (16.67\%), and expectantly in 210 patients (50\%). The difference in the rate of AEs in patients who underwent ERCP emergently or urgently compared to patients who underwent the procedure expectantly $(28.57 \%$ vs $19.05 \%, P=0.09)$ was not statistically significant ( $>$ Fig. 2 ). The difference in the rate of ERCP complications between patients who had the procedure done emergently compared to expectantly $(7.14 \%$ vs $4.76 \%, P=0.44$ ) was not statistically significant ( $\triangleright$ Fig. 2 ).

\section{Trauma}

Three hundred and twenty patients with trauma developed BDL and 150 patients (46.88\%) underwent ERCP. ERCP was performed emergently in 50 patients (33.3\%), urgently in 20 patients $(13.3 \%)$, and expectantly in 80 patients $(53.33 \%)$. The rate of AEs in patients who underwent ERCP emergently $(80 \%$ vs $62.5 \%, P=0.03)$ or urgently $(100 \%$ vs $62.5 \%, P=0.001)$ was higher compared to patients who underwent the procedure expectantly ( Fig. 3 ).

\section{Impact of ERCP technique}

Three hundred and fifty patients underwent ERCP intervention. Of them, 200 (57.14\%) had a biliary stent, 70 patients (20\%) had sphincterotomy, and 80 patients $(22.86 \%)$ had combination therapy. Combination therapy had a lower rate of ERCP failure compared to biliary stent ( $25 \%$ vs $12.5 \%, P=0.02$ ). There was no statistically significant difference in the rate of ERCPO failures with biliary stent and sphincterotomy (25\% vs $14.29 \%$, $P=0.06)$ or sphincterotomy and combination therapy $(14.29 \%$ vs $12.5 \%, P=0.74)$.

\section{Discussion}

We present a retrospective cohort study of 600 patients who underwent ERCP after BDL. We found that patients with BDL who had expectant ERCP had a lower risk of AEs, which is consistent with prior studies $[1,4]$. This supports the notion that ERCP does not need to be performed emergently or urgently. We also found that combination therapy had a lower incidence of ERCP failure compared to biliary stent placement alone, but that there was no statistical significance between combination therapy and sphincterotomy alone.

Four prior reports have investigated outcomes associated with the endoscopic technique and timing of BDLs [1,4-6]. These reports included 518, 1,028, 100, and 58 patients, respectively. In the study by Adler et al [1], there was no significant difference in post-ERCP AEs based on timing (21\% emergent, $22.9 \%$ urgent, $24.6 \%$ expectant), but the 90 -day mortality rate was lowest in the expectant group. The study by Abbas et al [4] also found a lower mortality rate and a lower rate of AEs among patients who underwent expectant ERCP.

The findings of our study are consistent with the findings of Abbas et al [4], which is that expectant ERCP has a lower rate of AEs. Abbas et al [4] cite a concern for "severity bias," which is that patients who are more ill at the time of presentation are more likely to undergo emergent ERCP, and hence, are also at higher risk of AEs. A similar pattern has been seen with outcomes regarding timing of endoscopic intervention in patients with gastrointestinal bleeding, which have been associated with higher rates of mortality in patients undergoing endoscopy on an emergent basis [7-11]. Our study had a greater percentage of AEs in patients undergoing ERCP for BDL. This could have been for several reasons, including that the Explorys database rounds patient populations to the closest 10 , which may have affected total population sizes. In addition, it is possible that some AEs were a consequence of the surgery itself and not the ERCP that was done afterward.

Prior studies assessing the technique of endoscopic intervention produced mixed results. Haidar et al [5] found that interventions involving stent placement were more successful than sphincterotomy alone. However, Chandra et al [6] found that sphincterotomy was as efficacious as stent placement, but sphincterotomy was faster and more cost-effective.

Our study found that combination therapy had a lower incidence of AEs compared to sphincterotomy and biliary stent placement alone; however, this did not reach statistical signifi- 
- Table 1 Baseline demographics in bile duct leak (BDL) patients who receiving endoscopic retrograde cholangiopancreatography (ERCP) based on procedure timing.

\begin{tabular}{|c|c|c|c|c|}
\hline Demographics & Emergent $(n=200)$ & Urgent $(n=100)$ & Expectant $(n=300)$ & $P$ value \\
\hline \multicolumn{5}{|l|}{ Age } \\
\hline . $>65$ & $70(35 \%)$ & $40(40 \%)$ & $120(40 \%)$ & \multirow[t]{3}{*}{0.19} \\
\hline - 45-65 & $80(40 \%)$ & $30(30 \%)$ & $90(30 \%)$ & \\
\hline . $<45$ & $50(25 \%)$ & $30(30 \%)$ & $90(30 \%)$ & \\
\hline \multicolumn{5}{|l|}{ Gender } \\
\hline - Male & $70(35 \%)$ & $40(40 \%)$ & $120(40 \%)$ & \multirow[t]{2}{*}{0.49} \\
\hline - Female & $130(65 \%)$ & $60(60 \%)$ & $180(60 \%)$ & \\
\hline \multicolumn{5}{|l|}{ Race } \\
\hline - White & $150(75 \%)$ & $80(80 \%)$ & $240(80 \%)$ & \multirow[t]{2}{*}{0.39} \\
\hline - African American & $30(15 \%)$ & $20(20 \%)$ & $40(13 \%)$ & \\
\hline \multicolumn{5}{|l|}{ Insurance } \\
\hline - Private & $100(50 \%)$ & $50(50 \%)$ & $160(53 \%)$ & \multirow[t]{3}{*}{0.80} \\
\hline - Medicare & $60(30 \%)$ & $30(30 \%)$ & $100(33 \%)$ & \\
\hline - Medicaid & $40(20 \%)$ & $20(20 \%)$ & $50(17 \%)$ & \\
\hline Tobacco abuse & $50(25 \%)$ & $20(20 \%)$ & $70(23 \%)$ & 0.62 \\
\hline Alcohol abuse & $10(5 \%)$ & $10(10 \%)$ & $20(7 \%)$ & 0.26 \\
\hline Obesity & $80(40 \%)$ & $40(40 \%)$ & $120(40 \%)$ & 1 \\
\hline HTN & $120(60 \%)$ & $60(60 \%)$ & $180(60 \%)$ & 1 \\
\hline HLD & $80(40 \%)$ & $40(40 \%)$ & $140(47 \%)$ & 0.25 \\
\hline DM & $60(30 \%)$ & $40(40 \%)$ & $90(30 \%)$ & 0.14 \\
\hline$C A D$ & $40(20 \%)$ & $20(20 \%)$ & $60(20 \%)$ & 1 \\
\hline $\mathrm{HF}$ & $40(20 \%)$ & $20(20 \%)$ & $50(17 \%)$ & 0.57 \\
\hline CKD & $40(20 \%)$ & $20(20 \%)$ & $50(17 \%)$ & 0.57 \\
\hline COPD & $40(20 \%)$ & $20(20 \%)$ & $60(20 \%)$ & 1 \\
\hline
\end{tabular}

cance. However, all three techniques had similar efficacy with regard to ERCP failure. Emergent ERCP had a higher risk of post-ERCP pancreatitis $(P=0.004)$.

Unique findings of our study are that patients with COPD had a higher risk of ERCP failure when the procedure was done emergently. This is suspected to be from decreased functional reserve and increased risk of complications with anesthesia in patients with chronic lung failure. We found that patients who underwent expectant ERCP were also significantly less likely to be re-hospitalized with bile peritonitis, abdominal abscess or persistent BDL within the first 3 months, which further emphasizes the benefit of expectant ERCP. We also found no difference with regard to outcomes based on patient demographic parameters. In addition, patients with a BDL from a traumatic injury have better outcomes if ERCP is performed expectantly.
No difference was seen with regard to timing in patients who had a BDL after cholecystectomy.

\section{Limitations}

The study was based on an administrative database analysis. There was a lack of information regarding grade of the biliary leaks (low vs high) and location of leaks because there were no available codes for this information. Because of the structure of the Explorys database, we were unable to determine the endpoint of leak resolution.

Because our study was database-driven using de-identified patient data, we were unable to assess for additional outcomes, such as the effect of admission over a weekend or need for biloma drainage or surgical intervention after endoscopy. We were also unable to assess several types of surgery leading to BDLs (e.g. liver resection, liver transplant, a Whipple procedure) or 


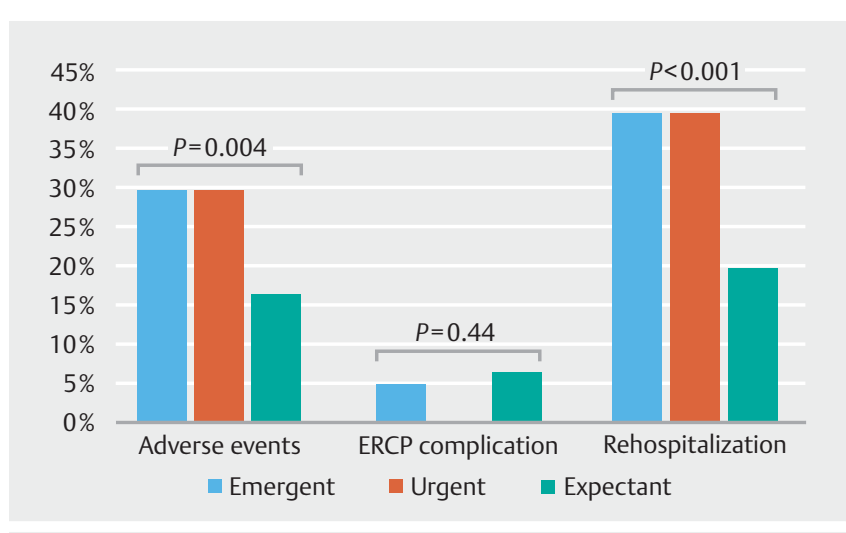

- Fig. 1 Comparison of various outcomes based on ERCP timing.

- Table 2 Baseline demographics in patients who had adverse events based on ERCP timing.

\begin{tabular}{|l|l|l|l|}
\hline $\begin{array}{l}\text { Demo- } \\
\text { graphics }\end{array}$ & $\begin{array}{l}\text { Emergent } \\
(\mathbf{n = 6 0})\end{array}$ & $\begin{array}{l}\text { Expectant } \\
(\mathbf{n = 5 0})\end{array}$ \\
\hline Age $>65$ & $20(33 \%)$ & $20(40 \%)$ & 0.44 \\
\hline Female & $30(50 \%)$ & $30(60 \%)$ & 0.29 \\
\hline White & $40(67 \%)$ & $40(80 \%)$ & 0.12 \\
\hline Private & $20(33 \%)$ & $20(40 \%)$ & 0.44 \\
\hline Medicare & $30(50 \%)$ & $20(40 \%)$ & 0.29 \\
\hline Obesity & $20(33 \%)$ & $20(40 \%)$ & 0.44 \\
\hline HTN & $40(67 \%)$ & $30(60 \%)$ & 0.44 \\
\hline HLD & $20(33 \%)$ & $20(40 \%)$ & 0.44 \\
\hline DM & $20(33 \%)$ & $20(40 \%)$ & 0.44 \\
\hline HF & $10(17 \%)$ & $10(20 \%)$ & 0.68 \\
\hline COPD & $10(17 \%)$ & 0 & 0.002 \\
\hline
\end{tabular}

HTN, hypertension, HLD, hyperlipidemia; diabetes mellitus; HF, heart failure; COPD, chronic obstructive pulmonary disease.

if there was any correlation between type of surgery/trauma and type of intervention. This is likely because of the small size of cohorts in the database, as a cohort of fewer than 10 patients is undetectable within the database. Due to this limitation, we were also unable to report numbers for each AE and complication. Due to a lack of ICD codes, we were unable to assess bile duct diameter or the location of the BDL (i.e. duct of Luschka, cystic duct stump, hepatic duct, biliary duct). Both duct diameter and location of BDL have been reported as possible associations $[1,4]$, but remain controversial.

The Explorys database [17] relies on the use of ICD diagnosis codes to identify patients in different cohorts. An inherent limitation of such databases includes verifying the diagnosis and criteria used to arrive at a diagnosis [18]. Further, the Explorys database is composed of population level data, so individual patient-level information cannot be obtained. As a result, beyond the surgeries that were included in our analysis, we were unable to ascertain the etiology of the BDL for the remainder of the pa-

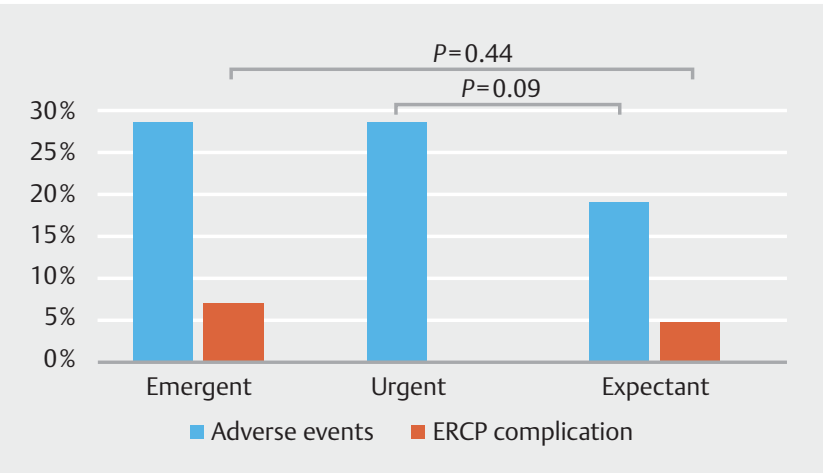

- Fig. 2 Comparison of adverse events and ERCP complication based on ERCP timing for post-cholecystectomy BDL.

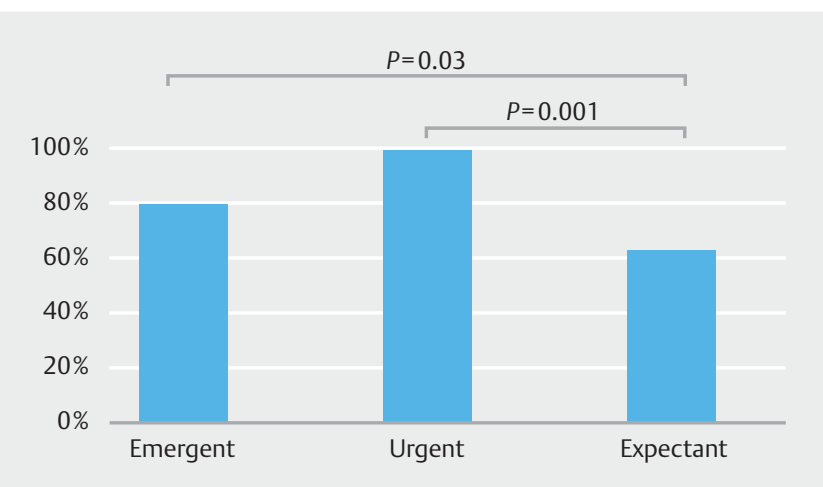

Fig. 3 Rate of adverse events based on ERCP timing in traumarelated $\mathrm{BDL}$.

tients. We were also unable to determine the treatments of patients who did not undergo ERCP or the number of patients who were managed conservatively. Another consequence of lack of patient-level data is the inability to delineate whether the AEs were an actual consequence of the ERCP procedure itself or a complication of the surgery causing the BDL. It is possible that patients who underwent emergent or urgent ERCP could have been more ill post-surgery and could be more prone to AEs from ERCP. Lack of patient-level data also precluded further subgroup analysis in the post-cholecystectomy group, where a common bile duct stone during cholecystectomy could have influenced early ERCP. Finally, the database does not allow for assessment of the type of bile duct stent that was used (plastic, metal, etc) so a comparison based on type of stent could not be made.

One of the major strengths of our study is that it is the second largest cohort of patients assessed with BDLs. Because we included patients from a national database, it is expected to represent the general population. This study also adds to current knowledge regarding the management of BDLs while also expanding on key aspects of managing patients with this condition. 


\section{Conclusion}

Standardized guidelines for effective management of BDLs are lacking [4]. Clinicians should be aware of the benefits of expectant timing of ERCP and that combination therapy has a lower incidence of ERCP failure compared to monotherapy with stenting alone.

\section{Competing interests}

The authors declare that they have no conflict of interest.

\section{References}

[1] Adler DG, Papachristou GI, Taylor LJ et al. Clinical outcomes in patients with bile leaks treated via ERCP with regard to the timing of ERCP: A large multicenter study. Gastrointest Endoscop 2017; 85: 766-772

[2] Vlaemynck K, Lahousse L, Vanlander A et al. Endoscopic management of biliary leaks: A systematic review with meta-analysis. Endoscopy 2019; 51: 1074-1081

[3] Rainio M, Lindström O, Udd M et al. Endoscopic therapy of biliary injury after cholecystectomy. Dig Dis Sci 2018; 63: 474-480

[4] Abbas A, Sethi S, Brady P et al. Endoscopic management of postcholecystectomy biliary leak: When and how? A nationwide study. Gastrointest Endoscop 2019; 90: 233-241

[5] Haidar H, Manasa E, Yassin K et al. Endoscopic treatment of postcholecystectomy bile leaks: a tertiary center experience. Surg Endosc 2020: doi:10.1007/s00464-020-07472-0

[6] Chandra S, Murali AR, Masadeh M et al. Comparison of biliary stent versus biliary sphincterotomy alone in the treatment of bile leak. Dig Dis 2020; 38: 32-37

[7] Tanaka M, Takahata S, Konomi H et al. Long-term consequence of endoscopic sphincterotomy for bile duct stones. Gastrointest Endosc 1998; 48: 465-469
[8] Sugiyama M, Atomi Y. Risk factors predictive of late complications after endoscopic sphincterotomy for bile duct stones: long-term (more than 10 years) follow-up study. Am J Gastroenterol 2002; 97: 2763-2767

[9] Pereira-Lima JC, Jakobs R, Winter UH et al. Long-term results (7 to 10 years) of endoscopic papillotomy for choledocholithiasis. Multivariate analysis of prognostic factors for the recurrence of biliary symptoms. Gastrointest Endosc 1998; 48: 457-464

[10] Sugiyama M, Atomi Y. Follow-up of more than 10 years after endoscopic sphincterotomy for choledocholithiasis in young patients. $\mathrm{Br}$ J Surg 1998; 85: 917-921

[11] Oliveira-Cunha M, Dennison AR, Garcea G. Late complications after endoscopic sphincterotomy. Surg Laparosc Endosc Percutan Tech 2016; $26: 1-5$

[12] Laursen SB, Leontiadis GI, Stanley AJ et al. Relationship between timing of endoscopy and mortality in patients with peptic ulcer bleeding: a nationwide cohort study. Gastrointest Endosc 2017; 85: 936-944

[13] Kumar NL, Cohen AJ, Nayor J et al. Timing of upper endoscopy influences outcomes in patients with acute nonvariceal upper GI bleeding. Gastrointest Endosc 2017; 85: 945-952

[14] Lau JYW, Yu Y, Tang RSY et al. Timing of endoscopy for acute upper gastrointestinal bleeding. N Engl J Med 2020; 382: 1299-1308

[15] Chathadi KV, Chandrasekhara V, Acosta RD et al. The role of ERCP in benign diseases of the biliary tract. Gastrointest Endosc 2015; 81: 795-803

[16] Dumonceau JM, Tringali A, Blero D et al. Biliary stenting: indications, choice of stents and results: European Society of Gastrointestinal Endoscopy (ESGE) clinical guideline. Endoscopy 2012; 44: 277-298

[17] Explorys team. We unlock the power of BIG DATA to improve healthcare for everyone. Available at (Accessed November 23, 2019): https://www.ibm.com/watson/health/about/

[18] Karb D, Mansoor E, Sravanthi Parasa GSC. Prevalence of diagnosed celiac disease and associated conditions in the United States between 2012-2017: Results form a national Electronic Patient Database. | Gastroenterol Hepatol Res 2019; 8: 2793-2799

\section{CORRECTION}

Aakash Desai, Patrick Twohig, Sophie Trujillo et al. Clinical efficacy, timing, and outcomes of ERCP for management of bile duct leaks: a nationwide cohort study

Endoscopy International Open 2021; 09: E247-E252.

DOI: $10.1055 / a-1322-2425$

In the above mentioned article a sentence in conclusion of abstract and article were incorrect. Correct is: "Combination therapy is superior to stenting but not sphincterotomy." and "Clinicians should be aware of the benefits of expectant timing of ERCP and that combination therapy has a lower incidence of ERCP failure compared to monotherapy with stenting alone." 